\title{
A potential impact of SARS-CoV-2 on pituitary glands and pituitary neuroendocrine tumors
}

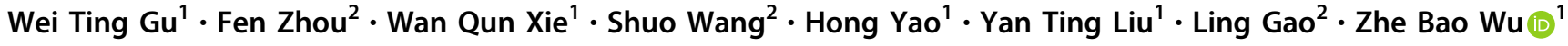

Received: 21 December 2020 / Accepted: 12 March 2021 / Published online: 30 March 2021

(c) The Author(s), under exclusive licence to Springer Science+Business Media, LLC, part of Springer Nature 2021

\begin{abstract}
Introduction Angiotensin-converting enzyme 2 (ACE2) is the receptor of severe acute respiratory syndrome coronavirus 2 (SARS-CoV-2). The effects of SARS-CoV-2 on normal pituitary glands function or pituitary neuroendocrine tumors (PitNETs) have not yet been elucidated. Thus, the present study aimed to investigate the potential risks of SARS-CoV-2 infection on the impairment of pituitary glands and the development of PitNETs.

Methods PitNETs tissues were obtained from 114 patients, and normal pituitary gland tissues were obtained from the autopsy. The mRNA levels of ACE2 and angiotensin II receptor type 1 (AGTR1) were examined by quantitative real-time PCR. Immunohistochemical staining was performed for ACE2 in 69 PitNETs and 3 normal pituitary glands. The primary tumor cells and pituitary cell lines (MMQ, GH3 and AtT-20/D16v-F2) were treated with diminazene aceturate (DIZE), an ACE2 agonist, with various dose regimens. The pituitary hormones between 43 patients with SARS-CoV-2 infection were compared with 45 healthy controls.

Results Pituitary glands and the majority of PitNET tissues showed low/negative ACE2 expression at both the mRNA and protein levels, while AGTR1 showed high expression in normal pituitary and corticotroph adenomas. ACE2 agonist increased the secretion of ACTH in AtT-20/D16v-F2 cells through downregulating AGTR1. The level of serum adrenocorticotropic hormone (ACTH) was significantly increased in COVID-19 patients compared to normal controls $(p<0.001)$, but was dramatically decreased in critical cases compared to non-critical patients $(p=0.003)$.

Conclusions This study revealed a potential impact of SARS-CoV-2 infection on corticotroph cells and adenomas.
\end{abstract}

Keywords Pituitary neuroendocrine tumors $\cdot$ Angiotensin-converting enzyme $2 \cdot$ Angiotensin II receptor type $1 \cdot$ Severe acute respiratory syndrome coronavirus $2 \cdot$ Pituitary gland

These authors contributed equally: Wei Ting Gu, Fen Zhou, Wan Qun Xie

Supplementary information The online version contains supplementary material available at https://doi.org/10.1007/s12020021-02697-y.

Ling Gao

ling.gao@whu.edu.cn

$\triangle$ Zhe Bao Wu

zhebaowu@aliyun.com

1 Department of Neurosurgery, Center of Pituitary Tumor, Ruijin Hospital, Shanghai Jiao Tong University School of Medicine, 200025 Shanghai, China

2 Department of Endocrinology \& Metabolism, Renmin Hospital of Wuhan University, 430060 Wuhan, China

\section{Introduction}

Coronavirus disease 2019 (COVID-19) is caused by severe acute respiratory syndrome coronavirus 2 (SARS-CoV-2). It has led to $>112,000,000$ infections and $>2,400,000$ deaths worldwide [1]. Presently, specific therapies preventing COVID-19 infection are lacking, which is essential to control the outbreak [2].

Angiotensin-converting enzyme 2 (ACE2) is the receptor of SARS virus and SARS-CoV-2 [3, 4]. It antagonizes the classical renin-angiotensin system (RAS) and exerts a protective effect against inflammation [5]. The expression and distribution of ACE2 indicate the risk and severity of COVID-19 [6]. Reportedly, ACE2 is expressed in the lung, heart, kidneys, and the central nervous system [7]. In the ACE2 transgenic mice model, the brain serves as a target organ for SARS infection $[8,9]$. However, whether ACE2 
is valuable for the prevention of SARS-CoV-2 infection is yet controversial [10].

The pituitary gland is the center of the endocrine system. Pituitary neuroendocrine tumors (PitNETs) are benign, originating from the anterior pituitary and accounting for $\sim 10-25 \%$ of all intracranial tumors. Although overexpression has been observed in several malignant tumors [11], the role of ACE2 on normal pituitary or PitNETs is yet to be elucidated.

Herein, we studied the potential risks of SARS-CoV-2 infection on pituitary glands and PitNETs. First, we explored the level of ACE2 in the normal pituitary glands and PitNETs, followed by the effect of ACE2 agonist over cellular proliferation and hormonal secretion of pituitary cell lines. Next, we assessed the changes in the pituitary function as a response to 2019-nCoV infection. Thus, the current study revealed that SARS-CoV-2 has a potential impact on corticotrophs.

\section{Materials and methods}

\section{Patients and tissue samples}

We performed a retrospective study involving 43 patients with COVID-19 as the study group, who were hospitalized in Renmin Hospital of Wuhan University from February 5 to Apri 5, 2020. All cases were laboratory-confirmed as SARSCoV-2 positive using quantitative RT-PCR (qRT-PCR) on nasal and pharyngeal swab specimens. The diagnosis of COVID-19 and the severity was determined according to the New Coronavirus Pneumonia Prevention and Control Program (7th edition) published by the National Health Commission of China. Briefly, patients with accompanying respiratory failure (mechanical ventilation needed), shock or multiple organ dysfunctions were diagnosed as critical type. The control group came from the population who previously received pituitary function evaluation and were classified as normal in the same center. 45 age - and gender-matched healthy controls were randomly selected and the data of their pituitary hormones were collected. Patients with a history of pituitary disease and/or renal insufficiency were excluded from the study. All the patients who underwent a complete pituitary hormone assessment and their matched-controls did not have a pituitary MRI.

A total of 114 PitNETs from a single expert center were collected. The cohort comprised of four normal pituitary tissues obtained from autopsy. This consecutive series encompassed PitNET types, including 24 (20.9\%) lactotrophs, 16 (13.9\%) somatotrophs, $13(11.3 \%)$ corticotrophs, 31 (27.0\%) gonadotrophs, $23(20.0 \%)$ null-cells, 4 (3.5\%) plurihormonal PIT1-positive PitNETs, 3 (2.6\%) mixed growth hormone/ prolactin (GH-PRL), and $1(0.9 \%)$ thyrotroph. The study was approved by the Ethical Review Board in Renmin Hospital of
Wuhan University and Ruijin Hospital of Shanghai Jiao Tong University School of Medicine.

\section{Pituitary hormone assessment}

In the study group, prolactin (PRL), somatotropin (GH), adrenocorticotropic hormone (ACTH), cortisol (8AM), thyroid stimulating hormone (TSH), free triiodothyronine (FT3), free thyroxine (FT4), luteinizing hormone (LH) and follicle stimulating hormone ( $\mathrm{FSH})$ were detected by electrochemiluminescent immunoassays according to the instructions from the manufacturer. In the control group, the data of serum PRL, GH, ACTH, cortisol, TSH, FT3, FT4, $\mathrm{LH}$ and FSH levels were retrieved from the dataset already kept in the same medical center. All the cases in the two groups had not received corticosteroid therapy or medications that could suppress TSH, ACTH and PRL within 5 days before pituitary hormone assessment.

\section{Public datasets acquisition and analysis}

RNA-seq data of normal tissues and PitNETs were downloaded from the Gene Expression Omnibus (GEO; https://www.ncbi.nlm.nih.gov/geo). 46 normal tissues, which included pituitary glands (5 tissues), distributed in different organs and 5 lactotroph adenomas were obtained for this study.

\section{Quantitative real-time PCR (qPCR)}

Total RNA of tumor tissues was extracted using TRIzol (Invitrogen, Carlsbad, CA, USA), following the manufacturer's instructions. $5 \mu \mathrm{g}$ RNA was reversely transcribed into cDNA using the cDNA Synthesis Kit (TaKaRa, Shiga, Japan). The mRNA expression level was quantified using qPCR with the SYBR Green real-time PCR Master Mix kit (TaKaRa Bio). PCR primers used were as follows: human ACE2 forward, 5'-CATTGGAGCAAGTGTTGGATCTT-3'; reverse: 5'-GAGCTAATGCATGCCATTCTCA-3'; human angiotensin II receptor type 1 (AGTR1) forward, 5'-GATGA TTGTCCCAAAGCTGG-3'; reverse, 5'-TAGGTAATTGCC AAAGGGCC-3'; human proto-oncogene receptor (MAS) forward, 5'- TTG TTG AGG AAC CCA CGA AC-3'; reverse, 5'- CCA CTG GGG AGA TGC TCA TA-3'; human $\beta$-actin forward, 5'-GGATGCAGAAGGAGATCACTG-3'; reverse: 5'-CGATCCACACGGAGTACTTG-3' [12-14]. The amplification reaction was carried out on an ABI PRISM 7500 Sequence Detection System (Applied Biosystems, Warrington, UK). The mRNA expression of the target genes was normalized to that of the endogenous reference gene $\beta$-actin and expressed as the fold-difference $(2-\Delta \Delta \mathrm{Ct})$. Low and high measurable levels were defined by ratio of specific/ beta-actin transcripts $<1$ and $\geq 1$, respectively [15]. 


\section{Immunohistochemistry (IHC) and scoring}

Tissue samples were fixed in $4 \%$ formalin, embedded in paraffin, and sliced into $4 \mathrm{~mm}$-thick sections. IHC was conducted using the Vectastain ABC Kit (Vector Laboratories, Burlingame, CA, USA). Rabbit primary antibody for ACE2 (1:200, ab108252) was purchased from Abcam (Cambridge, MA, USA). Finally, the slides were developed with DAB and counterstained with hematoxylin.

Slices were scanned by Pannoramic 1000 slide digitalization system (3DHISTECH, Budapest, Hungary). Images were captured at $\times 20$ magnification using CaseViewer 2.3(3DHISTECH, Budapest, Hungary). The field was selected with a good contrast of DAB chromogen and hematoxylin, which is considered region of interest. Before capturing the images, the color density and white balance were standardized for all images. All the acquired images were saved as JPEG format. After IHC images acquisition, ImageJ 1.48 version $(\mathrm{NIH}$, Bethesda, Maryland) analysis was performed and ACE2 immunostaining of pituitary glands and tumor tissues was scored as negative $(0+)$, low positive $(1+)$, positive $(2+)$ and high positive (3+), as described in the previous study [16].

\section{Cell culture and reagents}

Pituitary cell lines MMQ (CRL-10609 ${ }^{\mathrm{TM}}$ ), GH3 (CCL$82.1^{\mathrm{TM}}$ ), and AtT-20/D16v-F2 (AtT-20, CRL-1795 ${ }^{\mathrm{TM}}$ ) were purchased from the American Type Culture Collection (Manassas, USA). The MMQ and GH3 cell lines were cultured in Dulbecco's modified Eagle medium and F12 medium (Gibco, Grand Island, NY, USA), supplemented with $15 \%$ horse serum (Gibco) and $2.5 \%$ fetal bovine serum (FBS; Gibco). The AtT-20 cell line was cultured in RPMI1640 (Sangon Biotech, Shanghai, China) supplemented with $10 \%$ FBS (Gibco).

Primary human tumor cells were obtained from patients who underwent endoscopy surgery for PitNETs between March 2020 and August 2020 at the Department of Neurosurgery, Ruijin Hospital of Jiao tong University, Shanghai, China. As described in the previous study [17], the tumor cells were cultured in DMEM with $10 \%$ FBS and $100 \mathrm{U} / \mathrm{mL}$ penicillin/streptomycin (Gibco). Cells were seeded onto $10 \mathrm{~cm}$ dishes at a density of $10^{6}$ cells per well and were cultured for $48 \mathrm{~h}$ before treatment.

All cell lines and primary tumor cells were maintained in a humidified atmosphere with $5 \% \mathrm{CO}_{2}$ at $37{ }^{\circ} \mathrm{C}$. Diminazene aceturate (DIZE) was purchased from MedChemExpress (Shanghai, China).

\section{Establishment of stably transfected cells}

The recombinant plasmids (pCDH-puro-AGTR1) were constructed, and sequenced by Tongyong Biotechnologies
(Anhui, China). AtT-20 cell lines stably expressing DEPTOR or empty vectors, were constructed using the lentiviral technique. After $48 \mathrm{~h}$ transduction with lentiviral supernatant, cells were selected with $2 \mu \mathrm{g} / \mathrm{mL}$ puromycin for 1-2 weeks for stable transfectants.

\section{Western blotting}

Total proteins were extracted using the Total Protein Extraction Kit (Millipore Co., Billerica, MA, USA). The cultured tumor cells were lysed with RIPA lysis buffer (Beyotime, Shanghai, China). An equivalent of $30 \mathrm{mg}$ protein/sample was resolved by SDS-PAGE (Sangon Biotech, Shanghai, China) and transferred to polyvinylidene difluoride membranes (PVDF; Millipore). The membranes were blocked with $5 \%$ nonfat milk in Tris-buffered saline with $1 \%$ Tween-20 (TBST) buffer for $1 \mathrm{~h}$ and probed overnight with primary antibodies at $4{ }^{\circ} \mathrm{C}$. The antibodies used were as follows: ACE2 (ab108252, Abcam), and POMC (ab210605, Abcam), Tubulin (ab7291, Abcam) and AGTR1 (25343-1AP, Proteintech). The immunoreactive bands were detected using ECL detection reagent (Millipore) according to the manufacturer's instructions. Kidney paracancer tissues from two renal cell carcinoma patients were obtained for use as a positive control. Protein expression was quantified using ImageJ software (Wayne Rasband, National Institute of Mental Health, Bethesda, MD, USA).

\section{Cell proliferation assay}

Cell proliferation was measured using a WST-8 Cell Counting Kit-8 (CCK8) (Bimake, Houston, TX, USA) according to the manufacturer's instructions. MMQ, GH3, and AtT-20 and primary tumor cells were plated in 96-well plates at a density of $1 \times 10^{4}$ cells/well, respectively. All cells were treated with different concentrations of DIZE and assessed after $24 \mathrm{~h}, 48 \mathrm{~h}$ and $72 \mathrm{~h}$.

\section{Enzyme-linked immunosorbent assay (ELISA)}

The levels of the hormone in the cell culture supernatants were measured using ELISA kits (Signalway Antibody LLC, Maryland, MD, USA) at the indicated time points, with the appropriate treatment according to the manufacturer's recommendations.

\section{Statistical analysis}

All data were analyzed using GraphPad Prism, version 5 (GraphPad Software, La Jolla, CA, USA). The differences among categorical variables were analyzed using independent-sample Student's $t$ test or one-way analysis of variance (ANOVA). The immunoreactive scores of ACE2 
were analyzed using the non-parametric Kruskal-Wallis $\mathrm{H}$ test. $P<0.05$ indicated statistical significance.

\section{Results}

\section{Expression of ACE2 in human PitNETs and pituitary gland}

To identify whether the pituitary gland is a target organ of SARS-CoV-2, we evaluated the expression of ACE2 in human normal pituitary glands and PitNETs tissues. First, we obtained RNA-seq profile data of 42 normal tissues from GEO (GDS3834). Strikingly, ACE2 exhibited lower expression in the normal pituitary as compared to the other normal tissues. Then, Western blotting of eight tumor sections showed that the expression of ACE2 protein was lower in primary PitNET specimens (Supplementary Table 1) than that in renal tissue (Fig. 1a). To further confirm these clinical findings, 69 tumor sections from 114 PitNETs and 3 normal pituitary glands were stained by immunohistochemistry (IHC) (Fig. 1b, c); a low expression of ACE2 was detected in all normal pituitary glands. Moreover, among 69 samples of PitNET tissues, only $10(14.5 \%)$ samples showed positive ACE2 expression, while 59 (85.5\%) showed low- positive/ negative expression (Supplementary Table 2). However, no statistically significant differences $(p=0.589)$ were detected in ACE2 in pituitary glands or different PitNET subtypes. In addition, the examination in four human normal pituitary glands and 114 PitNETs tissues by realtime PCR (Fig. 1d and Supplementary Table 3) revealed that the overall expression of ACE2 was low but was relatively high in gonadotropic adenomas compared to normal pituitary and other subtypes of pituitary tumors $(p=0.016)$.

These data suggested that ACE2 is lowly expressed in pituitary glands and PitNETs at the protein and mRNA levels.

\section{Expression of RAS components in PitNETs}

The classical RAS ACE-Ang II-AGTR1 regulatory axis and the ACE2-Ang 1-7-MAS counter-regulatory axis played an essential role in maintaining homeostasis in humans (Fig. 1e). The entry of SARS-CoV-2 into cells is facilitated by the interaction between viral S-protein with the extracellular domains of the transmembrane ACE2 proteins, followed by subsequent downregulation of ACE2 expression and markedly elevated levels of circulating Angiotensin II (Ang II) [3, 18-21]. Ang II is converted to angiotensin 1-7 (Ang 1-7) by ACE2. Ang II binds to AGTR1, and Ang 1-7 binds to Mas. The expression of AGTR1 was increased in normal pituitary tissues compared to PitNETs $(0.004312 \pm 0.000748$ in normal pituitary glands and $0.001351 \pm 0.000136$ in PitNETs, $p<0.001)$. We also analyzed the correlation between the expression of AGTR1 and PitNET types and found that the expression of AGTR1 was increased in corticotroph adenomas compared to somatotroph adenomas $(p=0.016)$, gonadotroph adenomas $(p=0.014)$, and null-cell adenomas $(p=0.033)$. The expression of MAS was increased in normal pituitary tissues compared to PitNETs $(0.000889 \pm 0.000034$ in normal pituitary glands and $0.0005968 \pm 0.000020$ in PitNETs, $p=0.008$ ). However, no significant difference was observed among the PitNETs subtypes (Supplementary Table 4).

Next, we calculated the ratio of AGTR1 to MAS (AGTR1/MAS) in pituitary glands and PitNETs tissues. The AGTR1/MAS was increased in corticotroph adenomas and normal pituitary tissues compared to other subtypes of PitNETs $(p<0.001)$, while no significant difference was detected between corticotroph adenomas and normal pituitary tissues. These results suggested that corticotroph adenomas and normal pituitary tissues had higher mRNA levels of the ACE-Ang II-AGTR1 regulatory axis compared to other PitNETs subtypes.

\section{Function of ACE2 on the growth and hormone secretion of pituitary cells in vitro}

To clarify the role of ACE2 on different pituitary cells, we manipulated its function in pituitary MMQ, GH3, and AtT-20 cell lines. First, the expression of ACE2 was demonstrated in MMQ, GH3, and AtT-20 cells by immunoblotting (Fig. 2a). Next, we used DIZE to activate ACE2 in pituitary cells. CCK- 8 assays investigated the function of ACE2 on the proliferation of pituitary cell lines and primary tumor cells. The growth curve analysis revealed that DIZE does not inhibit the proliferation of pituitary cells at a dose of $100 \mu \mathrm{M}$ (Fig. 2b-j, Supplementary Fig. 1, and Supplementary Table 5). Next, ELISA was performed to examine the effect of ACE2 on the hormone secretion in GH3, MMQ, and AtT-20 cells (Fig. $2 \mathrm{k}-\mathrm{m}$ ). The activation of ACE2 by DIZE increased the ACTH secretion in AtT-20 cells; however, it did not show an obvious influence on the secretion of PRL in MMQ or GH3 cells, respectively. The effect of ACE2 on ACTH secretion was further explored by Western blot (Fig. 2n). Proopiomelanocortin (POMC) is the precursor for ACTH, which induces the synthesis and secretion of steroids from adrenal glands. The activation of ACE2 downregulates AGTR1 and upregulates POMC (Fig. 2n). In order to test whether AGTR1 decreased POMC expression in corticotroph, AtT-20 cells were transfected with AGTR1 overexpression plasmids, and POMC was examined by Western blotting. We found that the 
a

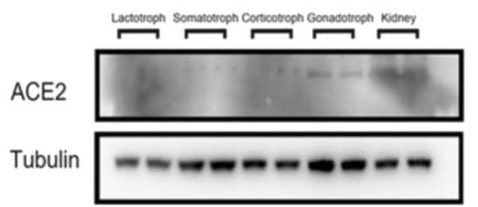

b
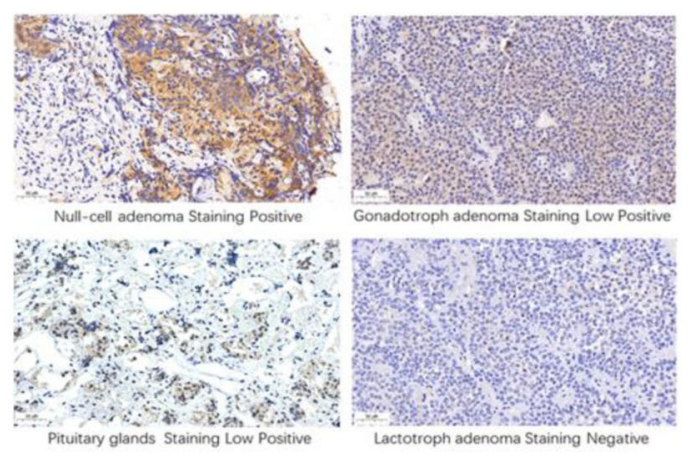

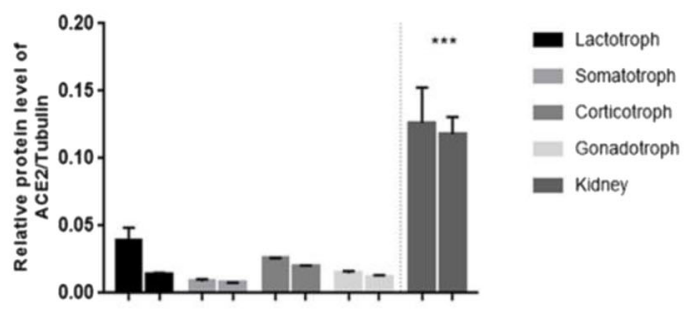

C

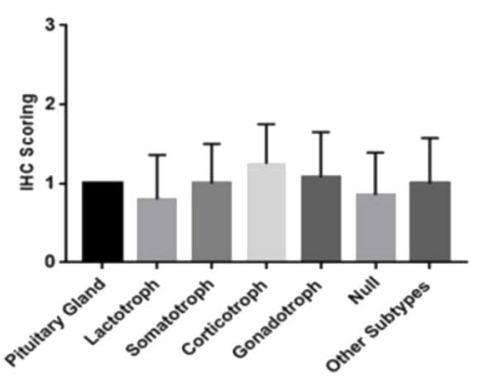

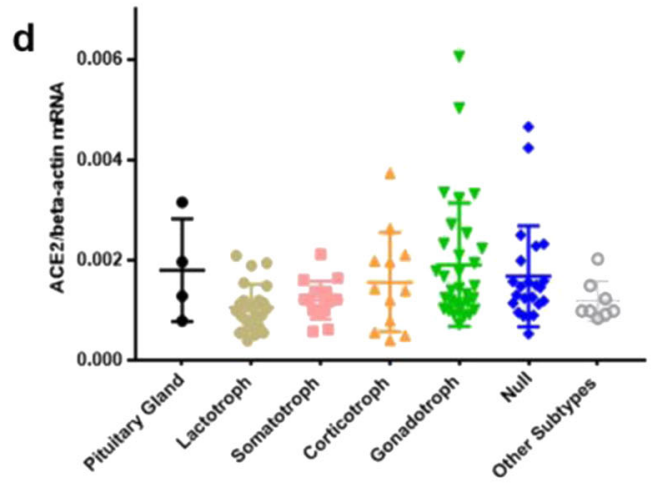

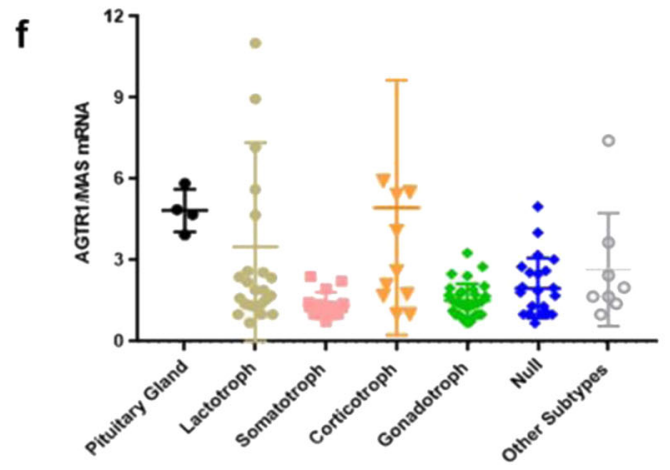

Fig. 1 The expression of ACE2 and RAS components in PitNETs and pituitary gland. a Western blot showed that ACE2 was low expressed in PitNETs compare to kidney tissues. b Representative immunostaining scores of ACE2 expressed in normal pituitary glands and PitNETs. Pituitary glands and PitNETs tissues was scored as negative $(0+)$, low positive $(1+)$, positive $(2+)$, and high positive $(3+)$, Scale bar, $50 \mu \mathrm{m}$. c Among 69 samples of PitNET tissues and 2 normal pituitary tissues, most samples showed low positive expression. No statistically significant differences $(p=0.589)$ were observed in ACE2 in pituitary glands or different PitNET subtypes. d Pituitary gland and PitNETs showed relatively low expression of ACE2 mRNA, which e

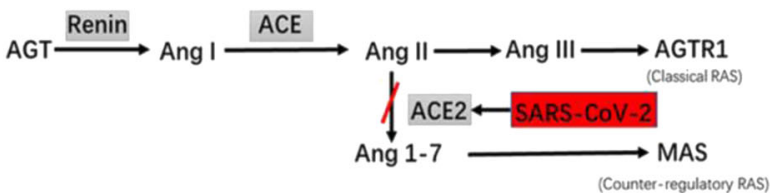

was otherwise relatively higher in gonadotroph adenomas $(P=0.016)$. Other Subtypes includes: 4 plurihormonal PIT1-positive PitNETs, 3 mixed growth hormone/prolactin (GH-PRL), and 1 thyrotroph. e Schematic illustration of RAS. f AGTR1/MAS in pituitary gland and PitNETs. AGTR1/MAS was otherwise higher in pituitary gland and corticotroph adenomas $(\mathrm{p} \ll 0.001)$. RAS renin-angiotensin system, AGT angiotensinogen, ACE angiotensin-converting enzyme, ACE2 angiotensin-converting enzyme 2, Ang I angiotensin I, Ang II angiotensin II, Ang III angiotensin III, Ang 1-7 angiotensin 1-7, Mas MAS proto-oncogene receptor, AGTR1 angiotensin II receptor type $1 .{ }^{*} P<$ $0.05 ; * * P<0.01 . * P<0.001$ 
a
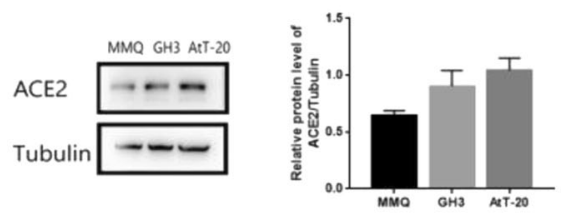

d

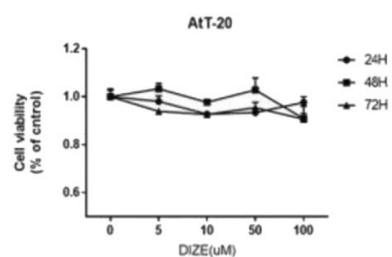

h
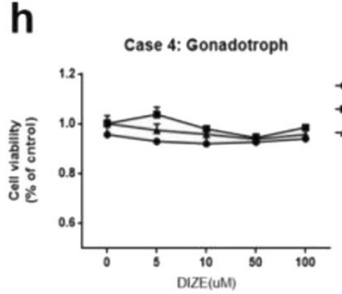

I

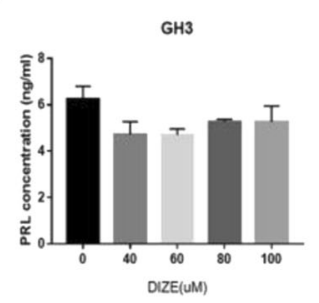

e

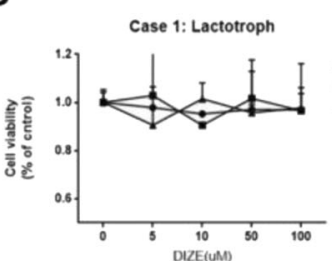

i

i

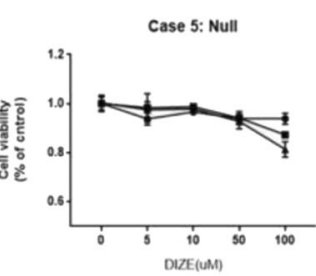

m

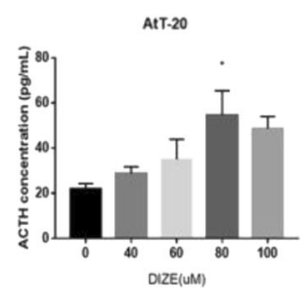

b

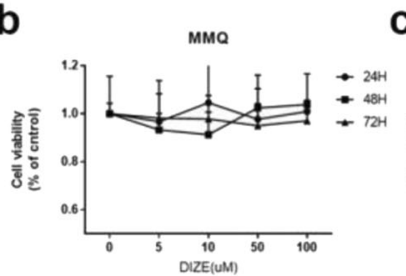

f

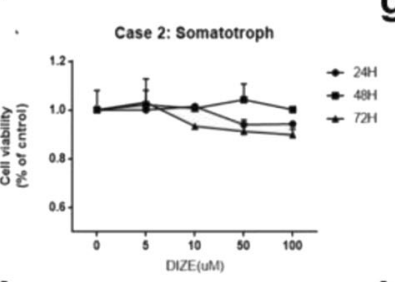

j

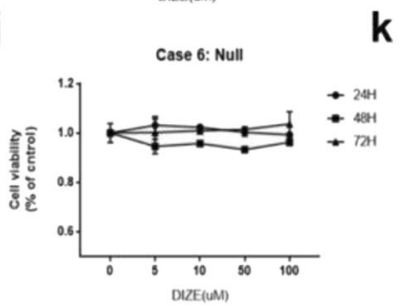

k

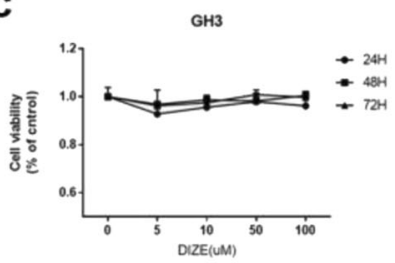

g
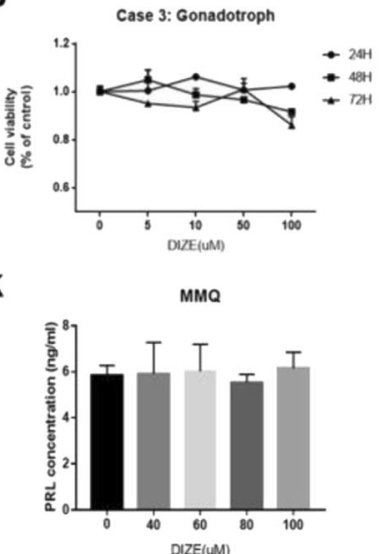

n
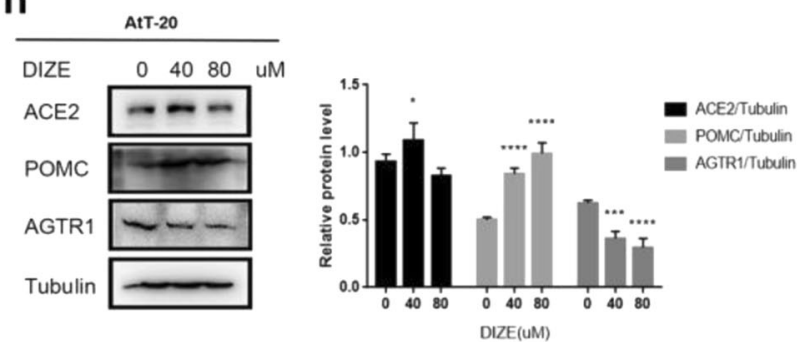

o
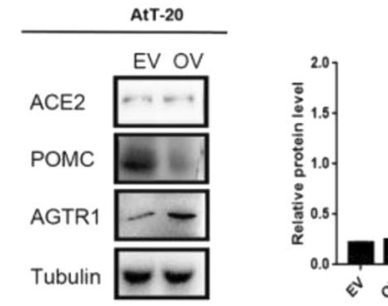

Fig. 2 Function of ACE2 on the hormone secretion of pituitary cells in vitro. a The expression of ACE2 in MMQ, GH3, and AtT-20/D16vF2(AtT-20) cells by western blot. b-j The function of ACE2 on the growth of pituitary cells in vitro. Pituitary cell lines (b-d) and primary pituitary tumor cells $(\mathbf{e}-\mathbf{j})$ were incubated with different concentrations of DIZE for 24, 48, and $72 \mathrm{~h}$, and cell proliferation was measured by CCK-8 assay. k-m MMQ cells, GH cells and AtT-20 cells were incubated with different concentrations of DIZE for $48 \mathrm{~h}$ and hormones secretion was detected by ELISA. $\mathbf{n}$ The effect of ACE2 was

activation of ACE2 elevated the POMC level and decreased AGTR1 expression, while AGTR1 overexpression exerted an opposite effects on POMC expression (Fig. 20).

Together, these data indicated that the activation of ACE2 by DIZE increases the ACTH secretion, but no difference was detected in PRL secretion and cell viability. confirmed by western blot. o AGTR1 overexpression had the opposite effects on ACTH secretion. PRL prolactin, ACTH adrenocorticotropic hormone, RAS renin-angiotensin system, DIZE diminazene aceturate, AGT angiotensinogen, ACE angiotensin-converting enzyme, ACE2 angiotensin-converting enzyme 2, Ang I angiotensin I, Ang II angiotensin II, Ang III angiotensin III, Ang 1-7 angiotensin 1-7, Mas MAS proto-oncogene receptor, AGTR1 angiotensin II receptor type 1, POMC proopiomelanocortin. $* P<0.05 ; * * P<0.01$. $* P<0.001$

\section{Pituitary function in COVID-19 patients}

To investigate the role of COVID-19 on pituitary function, ELISA was performed to detect the level of hormone secretion. Compared to the control group (Table 1), COVID-19 patients had significantly higher PRL (15.46 \pm $1.016 \mathrm{ng} / \mathrm{mL}$ vs. $11.34 \pm 1.184 \mathrm{ng} / \mathrm{mL}, \quad p=0.010)$ and 
Table 1 Hormone profiles in COVID-19 group and the control group

\begin{tabular}{lllr}
\hline Feature & $\begin{array}{l}\text { Patients with } \\
\text { COVID-19 }(n=43)\end{array}$ & $\begin{array}{l}\text { Healthy control } \\
(n=45)\end{array}$ & $p$ \\
\hline Age $(\mathrm{Y}):$ & $58.14 \pm 1.96$ & $53.2 \pm 1.85$ & 0.070 \\
Sex: & & & 0.355 \\
Female & $28(65.12 \%)$ & $25(55.96 \%)$ & \\
Male & $15(34.88 \%)$ & $20(44.44 \%)$ & \\
PRL $(\mathrm{ng} / \mathrm{mL})$ & $15.46 \pm 1.02$ & $11.34 \pm 1.18$ & 0.010 \\
GH $(\mathrm{ng} / \mathrm{mL})$ & $0.76 \pm 0.24$ & $0.50 \pm 0.13$ & 0.342 \\
ACTH $(\mathrm{pg} / \mathrm{mL})$ & $46.80 \pm 3.38$ & $28.34 \pm 1.77$ & $<0.001$ \\
Serum Cortisol & $17.60 \pm 1.39$ & $14.66 \pm 0.71$ & 0.059 \\
$(\mu \mathrm{g} / \mathrm{dL})$ & & & \\
FSH $(\mathrm{mIU} / \mathrm{mL})$ & $33.12 \pm 4.92$ & $27.06 \pm 3.94$ & 0.336 \\
LH $(\mathrm{mIU} / \mathrm{ml})$ & $16.47 \pm 2.372$ & $12.12 \pm 1.58$ & 0.127 \\
TSH $(\mu \mathrm{IU} / \mathrm{mL})$ & $2.13 \pm 0.22$ & $2.21 \pm 0.21$ & 0.787 \\
FT3 $(\mathrm{pg} / \mathrm{mL})$ & $3.11 \pm 0.11$ & $2.98 \pm 0.06$ & 0.269 \\
FT4 $(\mathrm{ng} / \mathrm{dL})$ & $1.16 \pm 0.03$ & $1.20 \pm 0.02$ & 0.392 \\
\hline
\end{tabular}

$P R L$ prolactin, $G H$ somatotropin, $A C T H$ adrenocorticotropic hormone, cortisol (8AM), TSH thyroid stimulating hormone, FT3 free triiodothyronine, FT4 free thyroxine, $L H$ luteinizing hormone, FSH follicle stimulating hormone

ACTH secretion $(46.8 \pm 3.383 \mathrm{pg} / \mathrm{mL}$ vs. $28.34 \pm 1.765 \mathrm{pg} /$ $\mathrm{mL}, p<0.0001)$. However, no statistically significant difference was detected in serum GH, cortisol, TSH, FT3, FT4, $\mathrm{LH}$, or FSH between the two groups. Interestingly, 6 (14.0\%) COVID-19 patients diagnosed as "critical cases" had significantly lower serum ACTH compared to the noncritical COVID-19 patients $(23.00 \pm 4.737 \mathrm{pg} / \mathrm{mL}$ vs. $50.66 \pm 3.47 \mathrm{pg} / \mathrm{mL}, p=0.003)$. Also, no significant difference was detected in the serum PRL, GH, cortisol, TSH, FT3, FT4, LH, or FSH between critical and non-critical patients (Table 2).

Taken together, these results suggested that ACTH was inhibited in critical COVID-19 patients, and SARS-CoV-2 might exert a potential impact on corticotroph via the ACE2 and AGTR1 proteins.

\section{Discussion}

Hitherto, few studies have evaluated to the expression of ACE2 in pituitary and PitNETs. The present study conducted real-time PCR, Western blot, and IHC and demonstrated low expression of ACE2 in pituitary glands and PitNETs. These findings indicated that the pituitary glands and PitNETs have a low susceptibility to SARS-CoV-2 infection.

Low ACE2 might reduce the entry of SARS-CoV-2 or local viral load or deteriorate the function of the organ under stress or fail to exert a protective role. Next, we compared the pituitary hormone profiles of COVID-19
Table 2 Hormone profiles in critical cases and non-critical cases

\begin{tabular}{llll}
\hline Feature & $\begin{array}{l}\text { Critical cases } \\
(n=6)\end{array}$ & $\begin{array}{l}\text { Non-Critical cases } \\
(n=37)\end{array}$ & $P$ \\
\hline Age $(Y):$ & $65.33 \pm 2.85$ & $56.97 \pm 2.18$ & 0.141 \\
Sex $(\%):$ & $3(50.00 \%)$ & $25(67.57 \%)$ & 0.413 \\
Female & $3(50.00 \%)$ & $12(32.43 \%)$ & \\
Male & $18.27 \pm 3.60$ & $15.01 \pm 1.03$ & 0.271 \\
PRL $(\mathrm{ng} / \mathrm{mL})$ & $0.56 \pm 0.30$ & $0.79 \pm 0.28$ & 0.746 \\
GH $(\mathrm{ng} / \mathrm{mL})$ & $23.00 \pm 4.74$ & $50.66 \pm 3.47$ & 0.003 \\
ACTH $(\mathrm{pg} / \mathrm{mL})$ & $22.49 \pm 7.63$ & $16.80 \pm 1.08$ & 0.158 \\
Serum Cortisol & & & \\
$(\mu \mathrm{g} / \mathrm{dL})$ & $19.44 \pm 9.13$ & $35.34 \pm 5.47$ & 0.267 \\
FSH $(\mathrm{mIU} / \mathrm{mL})$ & $11.44 \pm 4.90$ & $17.29 \pm 2.64$ & 0.399 \\
LH $(\mathrm{mIU} / \mathrm{ml})$ & $3.13 \pm 1.08$ & $1.97 \pm 0.18$ & 0.064 \\
TSH $(\mu \mathrm{IU} / \mathrm{mL})$ & $2.60 \pm 0.41$ & $3.20 \pm 0.11$ & 0.061 \\
FT3 $(\mathrm{pg} / \mathrm{mL})$ & $1.18 \pm 0.11$ & $1.16 \pm 0.03$ & 0.827 \\
FT4 $(\mathrm{ng} / \mathrm{dL})$ & &
\end{tabular}

$P R L$ prolactin, $G H$ somatotropin, $A C T H$ adrenocorticotropic hormone, cortisol (8AM), TSH thyroid stimulating hormone, FT3 free triiodothyronine, FT4 free thyroxine, $L H$ luteinizing hormone, FSH follicle stimulating hormone

patients and age- and gender-matched healthy controls in this study and observed a significant increase in serum PRL and ACTH in patients compared to healthy individuals. However, in critical cases, the ACTH level was dramatically decreased compared to that in non-critical patients. The secretion of PRL and ACTH could be influenced by multiple factors, such as diet, stress, and drugs. Based on similar PRL and cortisol levels in noncritical COVID-19 patients and critical COVID-19 patients, we inferred that the ACTH level decrease in critical COVID-19 patients could be attributed to dysfunction, such as the possible damage of corticotrophs instead of stress responses or feedback regulation of adrenal axis. Thus, the current data provided evidence that the hypothalamic-pituitary-adrenal (HPA) axis is activated in non-critical COVID-19 patients but suppressed in critical patients. Furthermore, DIZE increases ACTH secretion in vitro, which could serve as the potential therapy for critical COVID-19 patients [22-24].

Currently, the normal HPA axis response to SARS-CoV-2 is unknown. Both the HPA axis and the RAS are activated in response to stress in humans that maintain the organ function by modulating a multitude of homeostatic processes, including immune defense mechanisms, inflammation, and cellular metabolism [25, 26]. Although presented as independent systems, the HPA and RAS are highly interactive, sharing several endocrine factors, including $\mathrm{ACTH}$, vasopressin, Ang II, and aldosterone. ACE2 antagonizes the role of Ang II [27] that regulates the homeostasis system of RAS [28, 29]. The ACE-Ang II-AGTR1 axis has proinflammatory effects that 
might lead to acute lung injury or myocarditis, whereas the ACE2-Ang 1-7-Mas axis has anti-inflammatory effects $[30,31]$. We found that corticotroph adenomas and normal pituitary tissues had higher mRNA levels for genes of the ACE-Ang II-AGTR1 axis compared to other subtypes PitNETs. Although the current data showed that ACE2 was highly expressed in the gonadotroph, high expression of AGTR1 in the pituitary gland and corticotroph adenomas was also detected. We also found that activation of ACE2 increased ACTH secretion by upregulating POMC and downregulating AGTR1 expression, while AGTR1 overexpression had the opposite effects on POMC expression in AtT-20 cells. Since RAS could act at both local and systemic levels [32, 33], AGTR1 might be another key protein in COVID-19 infection patients.

According to the current results, the impact of SARSCoV-2 on HPA with/without pituitary disease should be considered. Since previous studies have indicated that glucocorticoid therapy was not beneficial to patients affected with SARS and MERS, and the guidelines of the World Health Organization (WHO) did not prescribe glucocorticoids $[34,35]$. The physiological stress doses of hydrocortisone could be administered [36]. Moreover, some of the patients with PitNets presented hypopituitarism, requiring an extra stress dose of glucocorticoid supplementation [36].

Nevertheless, the present study has some limitations, such as a lack of direct evidence from the tissue and blood samples of COVID-19 patients and viral infection of cell line experiments. In conclusion, the current study revealed a potential impact of SARS-CoV-2 on pituitary glands and corticotrophs. Therefore, the current findings might propose a prevention strategy for the disease in clinical practice.

\section{Data availability}

Some or all data, models, or code generated or used during the study are available from the corresponding author by request (List items).

Funding This work was supported by the Shanghai Municipal Science and Technology Commission 18XD1403400 (Z.B.W.), Program of Shanghai Academic Research Leader (Z.B.W.), and Shanghai Training and Support Program for Outstanding Young Medical Talents (Z.B.W.).

\section{Compliance with ethical standards}

Conflict of interest The authors declare no competing interests.

Informed consent Informed consent was obtained from all individual participants included in the study.

Publisher's note Springer Nature remains neutral with regard to jurisdictional claims in published maps and institutional affiliations.

\section{References}

1. C. Wang, P. Horby, F. Hayden, G. Gao, A novel coronavirus outbreak of global health concern. Lancet 395(10223), 470-473 (2020). https://doi.org/10.1016/S0140-6736(20)30185-9

2. J. Sanders, M. Monogue, T. Jodlowski, J. Cutrell, Pharmacologic Treatments for Coronavirus Disease 2019 (COVID-19): a review. Jama. (2020) https://doi.org/10.1001/jama.2020.6019. Online ahead of print

3. P. Zhou, X. Yang, X. Wang, B. Hu, L. Zhang, W. Zhang, H. Si, Y. Zhu, B. Li, C. Huang, H. Chen, J. Chen, Y. Luo, H. Guo, R. Jiang, M. Liu, Y. Chen, X. Shen, X. Wang, X. Zheng, K. Zhao, Q. Chen, F. Deng, L. Liu, B. Yan, F. Zhan, Y. Wang, G. Xiao, Z. Shi, A pneumonia outbreak associated with a new coronavirus of probable bat origin. Nature 579(7798), 270-273 (2020). https:// doi.org/10.1038/s41586-020-2012-7

4. R. Yan, Y. Zhang, Y. Li, L. Xia, Y. Guo, Q. Zhou, Structural basis for the recognition of SARS-CoV-2 by full-length human ACE2. Science 367(6485), 1444-1448 (2020). https://doi.org/10. $1126 /$ science.abb2762

5. X. Chen, P. Tummala, T. Olbrych, R. Alexander, R. Medford, Angiotensin II Induces Monocyte Chemoattractant Protein-1 Gene Expression in Rat Vascular Smooth Muscle Cells. Circ. Res. 83 (9), 952-959 (1998). https://doi.org/10.1161/01.RES.83.9.952

6. H. Xu, L. Zhong, J. Deng, J. Peng, H. Dan, X. Zeng, T. Li, Q. Chen, High expression of ACE2 receptor of 2019-nCoV on the epithelial cells of oral mucosa. Int. J. Oral Sci. 12(1), 8 (2020). https://doi.org/10.1038/s41368-020-0074-x

7. T. Komatsu, Y. Suzuki, J. Imai, S. Sugano, M. Hida, A. anigami, S. Muroi, Y. Yamada, K. Hanaoka, Molecular cloning, mRNA expression and chromosomal localization of mouse angiotensin-converting enzyme-related carboxypeptidase (mACE2). D N A Seq. 13(4), 217-220 (2002). https://doi.org/ $10.1080 / 1042517021000021608$

8. P. McCray, L.F. Pewe, C. Wohlford-Lenane, M. Hickey, L. Manzel, L. Shi, J. Netland, H. Jia, C. Halabi, C. Sigmund, D. Meyerholz, P. Kirby, D. Look, S. Perlman, Lethal infection of K18-hACE2 mice infected with severe acute respiratory syndrome coronavirus. J. Virol. 81(2), 813-821 (2007). https://doi.org/10. 1128/JVI.02012-06

9. J. Netland, D. Meyerholz, S. Moore, M. Cassell, S. Perlman, Severe acute respiratory syndrome coronavirus infection causes neuronal death in the absence of encephalitis in mice transgenic for human ACE2. J. Virol. 82(15), 7264-7275 (2008). https://doi. org/10.1128/JVI.00737-08

10. H. Cheng, Y. Wang, G. Wang, Organ-protective effect of angiotensin-converting enzyme 2 and its effect on the prognosis of COVID-19. J. Med. Virol. (2020) https://doi.org/10.1002/jmv.25785

11. P. Chai, J. Yu, S. Ge, R. Jia, X. Fan, Genetic alteration, RNA expression, and DNA methylation profiling of coronavirus disease 2019 (COVID-19) receptor ACE2 in malignancies: a pan-cancer analysis. J. Hematol. Oncol. 13(1), 43 (2020). https://doi.org/10. 1186/s13045-020-00883-5

12. X. Fu, R. Lin, Y. Qiu, P. Yu, B. Lei, Overexpression of Angiotensin-Converting Enzyme 2 Ameliorates Amyloid betaInduced Inflammatory Response in Human Primary Retinal Pigment Epithelium. Investig. Ophthalmol. Vis. Sci. 58(7), 3018-3028 (2017). https://doi.org/10.1167/iovs.17-21546

13. N. Takahiro, C. Fumihisa, N. Takehiro, H. Chuyu, K. Akiko, S. Kaori, N. Takuo, A. Hiromitsu, I. Go, K. Atsushi, Y. Tatsuo, K. Kei, The Expression of MAS1, an Angiotensin (1-7) Receptor, in the Eutopic Proliferative Endometria of Endometriosis Patients. Gynecol. Obstet. Investig. 83(6), 600-607 (2018). https://doi.org/ $10.1159 / 000490561$ 
14. Y. Ma, Z. Xia, C. Ye, C. Lu, S. Zhou, J. Pan, C. Liu, J. Zhang, T. Liu, T. Hu, L. Xie, G. Wu, Y. Zhao, AGTR1 promotes lymph node metastasis in breast cancer by upregulating CXCR4/SDF-1 $\alpha$ and inducing cell migration and invasion. Aging (Albany NY) 11 (12), 3969-3992 (2019). https://doi.org/10.18632/aging.102032

15. E. Ferretti, D. Di Stefano, F. Zazzeroni, R. Gallo, A. Fratticci, R. Carfagnini, S. Angiulli, A. Santoro, G. Minniti, G. Tamburrano, E. Alesse, G. Cantore, A. Gulino, M. Jaffrain-Rea, Human pituitary tumours express the bHLH transcription factors NeuroD1 and ASH1. J Endocrinol. Investig. 26(10), 957-965 (2003). https://doi.org/10.1007/BF03348192

16. D. Mane, A. Kale, C. Belaldavar, Validation of immunoexpression of tenascin-C in oral precancerous and cancerous tissues using ImageJ analysis with novel immunohistochemistry profiler plugin: an immunohistochemical quantitative analysis. J. Oral Maxillofac. Pathol. 21(2), 211-217 (2017). https://doi.org/10. 4103/jomfp.JOMFP_234_16

17. S.J. Lin, Z.R. Wu, L. Cao, Y. Zhang, Z.G. Leng, Y.H. Guo, H.B. Shang, W.G. Zhao, X. Zhang, Z.B. Wu, Pituitary Tumor Suppression by Combination of Cabergoline and Chloroquine. J. Clin. Endocrinol. Metab. 102(10), 3692-3703 (2017).

18. A.C. Walls, Y.J. Park, M.A. Tortorici, A. Wall, A.T. McGuire, D. Veesler, Structure, Function, and Antigenicity of the SARS-CoV2 Spike Glycoprotein. Cell 181(2), 281-292.e6 (2020).

19. Y. Imai, K. Kuba, S. Rao, Y. Huan, F. Guo, B. Guan, P. Yang, R. Sarao, T. Wada, H. Leong-Poi, M. Crackower, A. Fukamizu, C. Hui, L. Hein, S. Uhlig, A. Slutsky, C. Jiang, J. Penninger, Angiotensinconverting enzyme 2 protects from severe acute lung failure. Nature 436(7047), 112-116 (2005). https://doi.org/10.1038/nature03712

20. K. Wang, M. Gheblawi, G.Y. Oudit, Angiotensin Converting Enzyme 2: A Double-Edged Sword. Circulation (2020). https:// doi.org/10.1161/CIRCULATIONAHA.120.047049

21. Y. Liu, Y. Yang, C. Zhang, F. Huang, F. Wang, J. Yuan, Z. Wang, J. Li, J. Li, C. Feng, Z. Zhang, L. Wang, L. Peng, L. Chen, - Fau, D. Bennett, Y. Qin, D. Zhao, S. Tan, L. Yin, J. Xu, C. Zhou, C. Jiang, L. Liu, Clinical and biochemical indexes from 2019-nCoV infected patients linked to viral loads and lung injury. Sci. China Life Sci. 63(3), 364-374 (2020). https://doi.org/10. 1007/s11427-020-1643-8

22. Y. Li, W. Zhou, L. Yang, R. You, Physiological and pathological regulation of ACE2, the SARS-CoV-2 receptor. Pharmacol Res. 157, 104833 (2020). https://doi.org/10.1016/j.phrs.2020.104833

23. C. Castardeli, C.L. Sartório, E.B. Pimentel, L. Forechi, J.G. Mill, The ACE 2 activator diminazene aceturate (DIZE) improves left ventricular diastolic dysfunction following myocardial infarction in rats. Biomed. Pharmacother. 107, 212-218 (2018)

24. L. Kangussu, T. de Almeida, T. Prestes, M. de Andrade De Maria, R. da Silva Filha, M. Vieira, A. Silva, A. Ferreira, Beneficial Effects of the Angiotensin-Converting Enzyme 2 Activator Dize in Renovascular Hypertension. Protein Pept. Lett. 26(7), 523-531 (2019). https://doi.org/10.2174/0929866526666190405123422

25. M.A. Flierl, D. Rittirsch, S. Weckbach, M. Huber-Lang, K. Ipaktchi, P.A. Ward, P.F. Stahel, Disturbances of the hypothalamic-pituitary-adrenal axis and plasma electrolytes during experimental sepsis. Ann. Intensive Care 1, 53 (2011). https://doi.org/10.1186/2110-5820-1-53

26. A. Polito, J. Aboab, D. Fau - Annane, D. Annane, The hypothalamic pituitary adrenal axis in sepsis. Novartis Found, Symp. 280,182-99; discussion 199-203 (2007).

27. M. Gheblawi, K. Wang, A. Viveiros, Q. Nguyen, J.C. Zhong, A.J. Turner, M.K. Raizada, M.B. Grant, G.Y. Oudit, AngiotensinConverting Enzyme 2: SARS-CoV-2 Receptor and Regulator of the Renin-Angiotensin System: Celebrating the 20th Anniversary of the Discovery of ACE2. Circ. Res. 126(10), 1456-1474 (2020). https://doi.org/10.1161/circresaha.120.317015

28. C. Tikellis, S. Bernardi, C. Wendy, W. Burns, Angiotensinconverting enzyme 2 is a key modulator of the renin-angiotensin system in cardiovascular and renal disease. Curr. Opin. Nephrol. Hypertens. 20(1), 62-68 (2011)

29. S. Forrester, G. Booz, C. Sigmund, T. Coffman, T. Kawai, V. Rizzo, R. Scalia, S. Eguchi, Angiotensin II Signal Transduction: an Update on Mechanisms of Physiology and Pathophysiology. Physiol. Rev. 1 98(3), 1627-1738 (2018)

30. H. Zhang, A. Baker, Recombinant human ACE2: acing out angiotensin II in ARDS therapy. Crit. Care 21(1), 305 (2017). https://doi.org/10.1186/s13054-017-1882-z

31. T.C. Hanff, M.O. Harhay, T.S. Brown, J.B. Cohen, A.M. Mohareb, Is There an Association Between COVID-19 Mortality and the Renin-Angiotensin System? A Call for Epidemiologic Investigations. Clin. Infect. Dis. 71(15), 870-874 (2020). https:// doi.org/10.1093/cid/ciaa329

32. K. Philipp, Y. Minghao, W. Jan, M. Christoph, K. Syed, B. Daniel, Angiotensin-converting enzyme 2-independent action of presumed angiotensin-converting enzyme 2 activators: studies in vivo, ex vivo, and in vitro. Hypertension 63(4), 774-778 (2014). https://doi.org/10. 1161/HYPERTENSIONAHA.113.02856

33. G. Pironti, R. Strachan, D. Abraham, S. Mon-Wei Yu, M. Chen, W. Chen, K. Hanada, L. Mao, L. Watson, H. Rockman, Circulating Exosomes Induced by Cardiac Pressure Overload Contain Functional Angiotensin II Type 1 Receptors. Circulation 131(24), 2120-2130 (2015). https://doi.org/10.1161/CIRCULATION AHA.115.015687

34. Y. Arabi, Y. Mandourah, F. Al-Hameed, A. Sindi, G. Almekhlafi, M. Hussein, J. Jose, R. Pinto, A. Al-Omari, A. Kharaba, A. Almotairi, K. Al Khatib, B. Alraddadi, S. Shalhoub, A. Abdulmomen, I. Qushmaq, A. Mady, O. Solaiman, A. Al-Aithan, R. AlRaddadi, A. Ragab, H. Balkhy, A. Al Harthy, A. Deeb, H. Al Mutairi, A. Al-Dawood, L. Merson, F. Hayden, R. Fowler, Corticosteroid Therapy for Critically Ill Patients with Middle East Respiratory Syndrome. Am. J. Respir. Crit. Care Med. 15, 700-701 (2018)

35. World Health O. Clinical management of severe acute respiratory infection when novel coronavirus (2019-nCoV) infection is suspected: interim guidance, 28 January 2020. (World Health Organization, Geneva), 2020)

36. U. Kaiser, R. Mirmira, P. Stewart, Our Response to COVID-19 as Endocrinologists and Diabetologists. J. Clin. Endocrinol. Metab. 105(5), dgaa148 (2020). https://doi.org/10.1210/clinem/dgaa148 